\title{
Scintigraphic Evidence of Improvement in Myocardial Perfusion Associated with Improvement in Physical Fitness in a patient with Angina
}

\author{
Maj Peter Lynch \\ MRCP, RAMC \\ Col I C Crawford \\ CBE, FRCP, L/RAMC \\ Queen Elizabeth Military Hospital, Woolwich
}

SUMMARY: A young soldier with a small myocardial infarction and a large area of ischaemic myocardiun? was followed up by maximal exercise testing and thallium scanning during a 15 month period of cardia $\overline{0}$ rehabilitation which included strenuous physical exercise. The area of ischemic myocardium was seen to reduce्巳 markedly over this period as measured by thallium scanning. Such clearly evident changes have not so far. been reported in these circumstances.

\section{Introduction}

While physical training appears to improve angina pectoris by increasing exercise tolerance ${ }^{1}$ and morale 2,3 and by reducing the amount of ST segment depression on the exercise electrocardiogram $^{4}$ it is not yet clear whether this is accompanied by an improvement in myocardial perfusion in the ischemic area. We have followed the course of a young soldier with angina pectoris by means of maximal exercise testing and thallium $\left(20 \mathrm{I}_{\mathrm{T}}\right)$ scanning and present evidence that the area of myocardium at risk has greatly diminished following improvement in physical fitness.

\section{Case Report}

The patient, a 35 year old cook, was admitted as an emergency with burning chest pain in a band across his chest, associated with tingling in the left arm which had come on at rest and lasted several hours. He had similar but milder pains for three weeks prior to admission which came on predictably while rushing uphill, but never at other times, and was relieved within 10 minutes by resting. The initial electrocardiogram (ECG) showed $\mathrm{T}$ wave inversion in standard lead $I$, and in lead V3. Over the next two weeks the $T$ wave normalised in standard lead I but remained unchanged in lead V3. Lactic dehydrogenase estimations on days one to three were 230,250 and 141 units respectively (normal range 36-188 units). On maximal exercise testing three weeks later the patient reached a maximal heart rate of 180 beats per minute, nine minutes into the Bruce protocol ${ }^{5}$ (stage III). The systolic blood pressure rose to $190 \mathrm{~mm} \mathrm{Hg}$.

Chest pain began at four minutes and was gone by 18 minutes, but the ECG did not change. Then lium scan carried out within 10 minutes of exero cise and again four hours later showed an extensitve and reversible filling defect in the septum (fig Three months later a further maximal exercise est and thallium scan was carried out. In the interine the patient had been given instructions to increasse his activity to the onset of pain, and reported thate he was now performing three half-hour sessi⿺辶卬 per week of circuit training in the gymnasium was walking eight miles every day. He was jogging intermittently. This time he exercised to 13 minutes of the Bruce protocol (stage IV) and reache a heart rate of 190 beats per minute, and a maximun $\$$ systolic blood pressure of $180 \mathrm{~mm} \mathrm{Hg}$. Chest paif began in the fifth minute and lessened as the test went on, to disappear by the fourteenth minute? Thallium scan showed a marked diminution of the ischemic area (fig 2). Six months later he under went cardiac catheterisation having been admitte twice in the interim with transient chest pain and palpitations without further abnormality being detected. Cardiac catheterisation showed total occlue sion of the anterior descending branch of the lef coronary artery distal to a large first diagonal and small first septal branch. Cross filling from the right coronary artery was noted. There was some dilation of the left ventricle (end-diastolic volume index $110 \mathrm{mls} / \mathrm{M}^{2}$ ) and apical hypokinesia but the ejection fraction remained good at 0.67 . Several months later? almost a year after his initial admission, the patient underwent a three week in-patient course of cardias rehabilitation which included three 20 minute exercise sessions daily to a heart rate $80 \%$ of thap reached on maximal exercise testing. Finally, 150 
ANTERIOR 18.12 .79

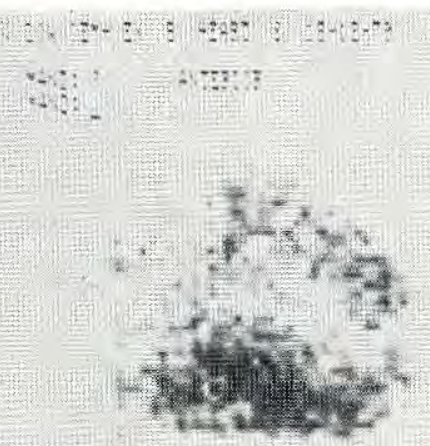

LAO $45 \quad 18.12 .79$
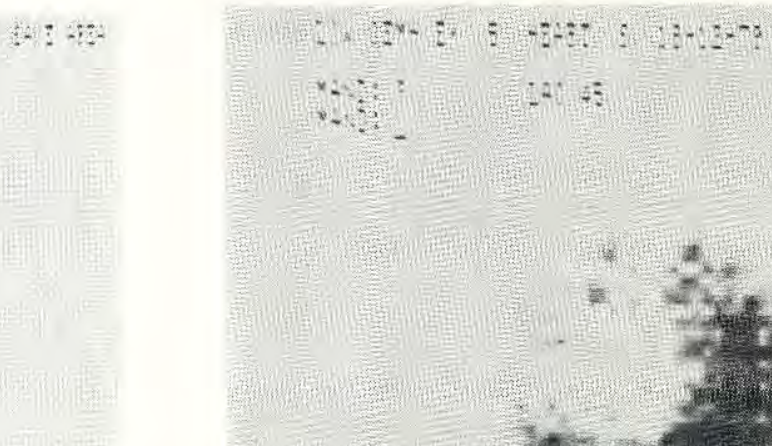

$x_{2}^{2}=2 \quad-24=5$

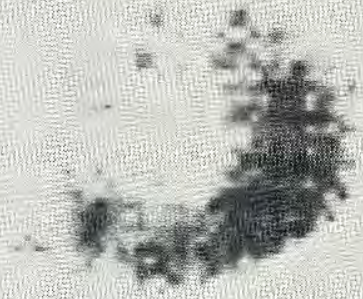

Fig. 1a. Exercise Views

ANTERIOR $\quad 18.12 .79$

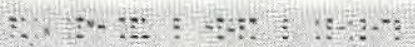

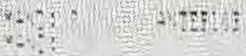

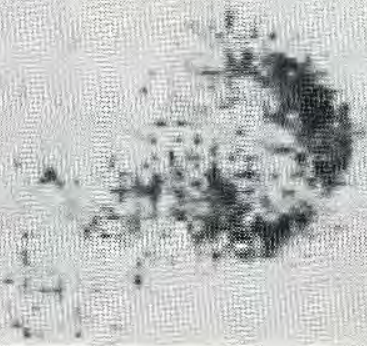

LAO $45 \quad 18.12 .79$

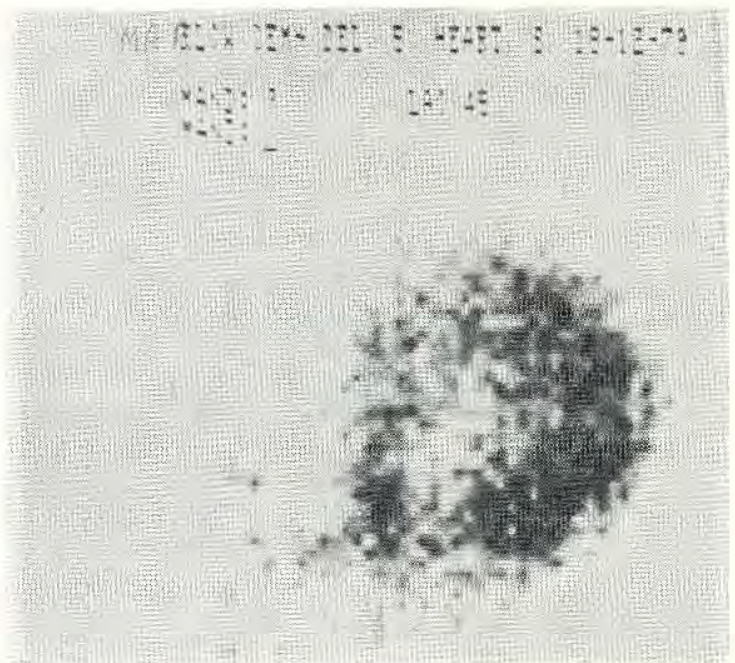

Fig. 1b Delayed Views

months after his initial event, he again underwent maximal exercise testing and thallium scanning. This time he exercised for 10 minutes to stage IV of the Bruce protocol and reached a heart rate of 190 beats per minute, and a maximum systolic blood pressure of $180 \mathrm{mmHg}$. Chest pain was present for 30 seconds in the seventh minute only, and again the ECG did not change. Thallium scan this time showed only a small defect at the apex (fig 3).

\section{Discussion}

The initial presentation of the patient suggested a small myocardial infarction or episode of coronary artery spasm. The ECG and enzyme findings, and later the left ventricular angiogram, were compatible. The angiographic findings in the coronary arteries suggested the patient had a slowly occluding major stenosis of the anterior descending branch of the left coronary artery. The event he suffered may have been the moment of complete occlusion or an embolic phenomenon. The anterior views of the three resting thallium scans suggest permanent underperfusion of the apex and are compatible with a small infarct in that area. Three weeks after the event the patient had a large reversible defect in the interventricular septum and had compatible 
ANTERIOR $\mathbf{7 . 2 . 8 0}$

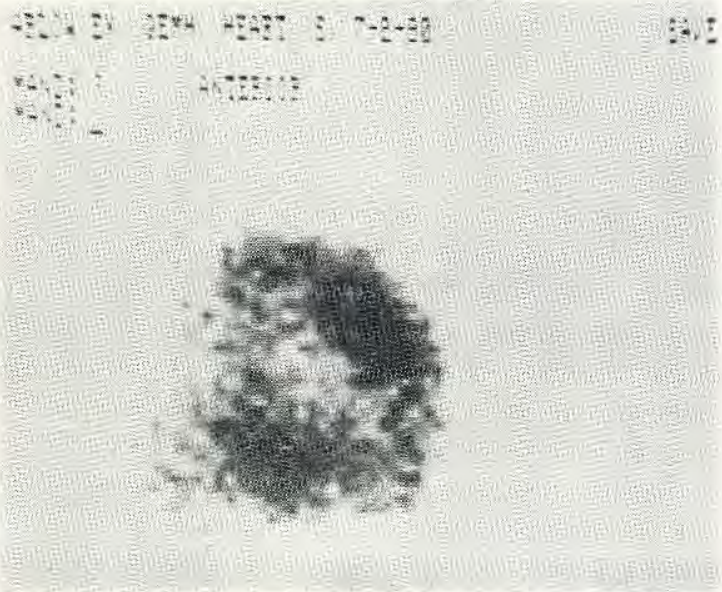

LAO $45 \quad 7.2 .80$
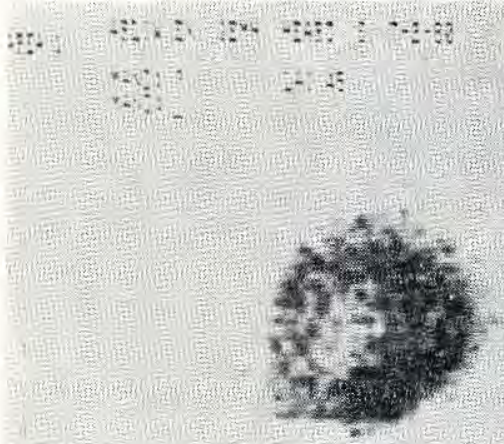

Fig. 2a Exercise Views
LAO $45 \quad 7.2 .80$
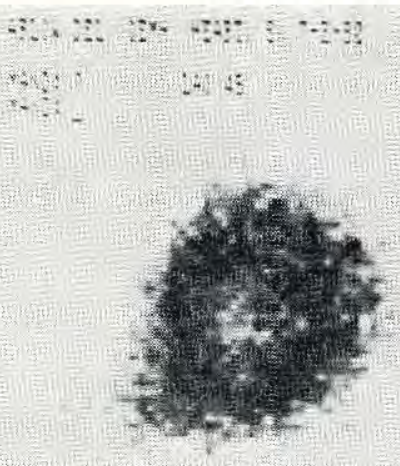

ANTERIOR $\quad \mathbf{7 . 2 . 8 0}$
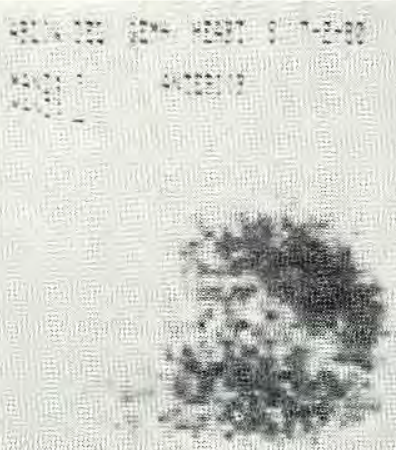

in: : -4 :

Fig. 2b Delayed Views symptoms. Over the next 15 months that area markedly reduced, as did the symptoms. This in itself is an important phenomenon and, to our knowledge, has never before been so clearly demonstrated. The fact that the period of improvement coincided with a change in lifestyle which included a marked increase in physical fitness in no way proves that the improvement was the result of exercise. Nevertheless, this association has been clearly shown in animal experiments ${ }^{6,}, 7,8$. There have been many attempts to investigate the phenomenon of improvement in angina with improvement in physical fitness. Most of these have concluded that peripheral factors, such as reduction of pulse rate and blood pressure for a given work load ${ }^{9-11}$, and increased mitochondrial efficiency in the peripheral muscles $^{12}$ are responsible. Nevertheless, there have been findings that the ratio of myocardial capillary to fibre ratio is increased ${ }^{13}$, that the bore of the coronary arteries is increased ${ }^{14}$, and other support ing evidence ${ }^{4}$. Froelicher et $\mathrm{al}^{15}$ presented 5 casesक with coronary artery disease in which a programme of strenuous physical exercise appcared to reduce? the area of ischemic myocardium but only one of 3 . these cases was reputed to have angina pectoris and in any case the changes were described by the 3 authors as "subtle."

We have shown that marked reduction in the areao of myocardium at risk in angina pectoris can occur That this reduction occurred in relation to strenuous 0 physical exercise is an indication that the improve-N ment in angina consequent on improved physicaln fitness may not be a peripheral phenomenon entirely. 
ANTERIOR $\quad 4.3 .81$

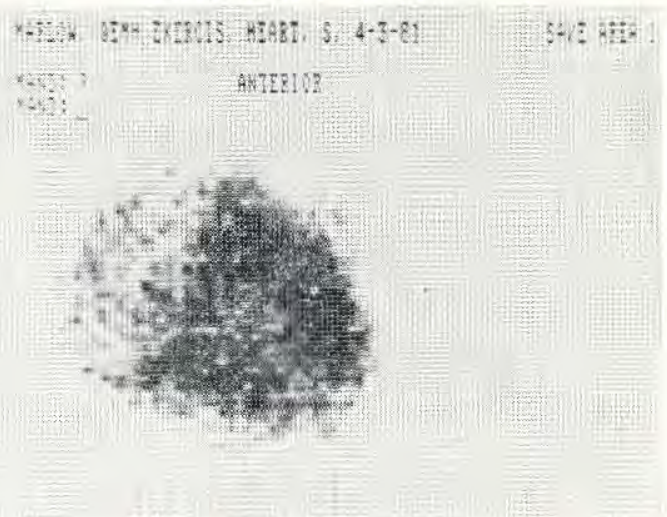

LAO $45 \quad 4.3 .81$

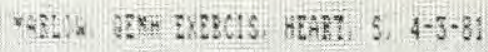

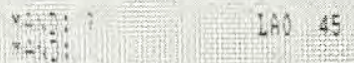

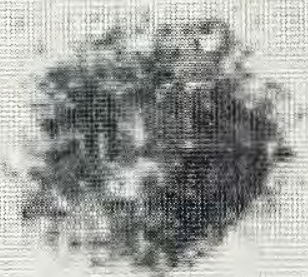

Fig. 3a Exercise Views

ANTERIOR 4.3 .81

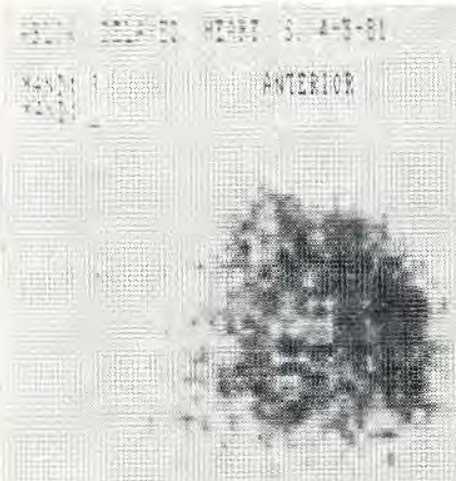

LAO $45 \quad 4.3 .81$

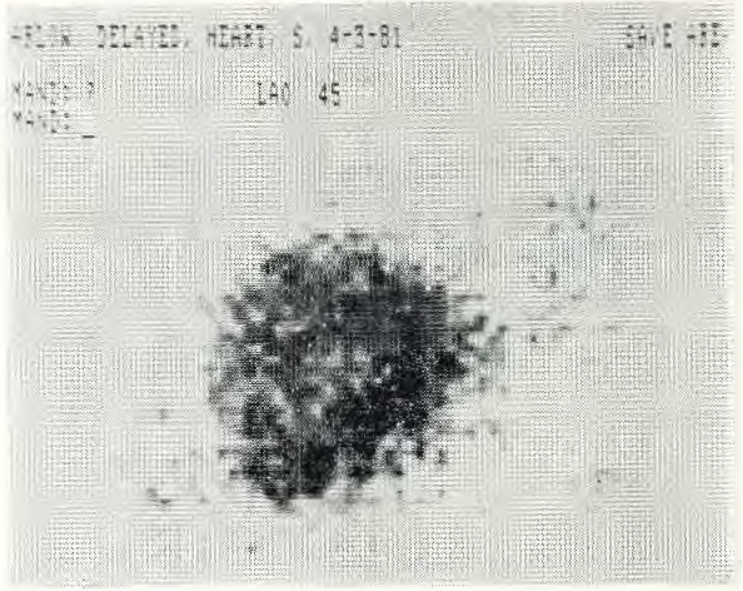

Fig. 3b Delayed Views

\section{REFERENCES}

1 SIM D N, NeILL W A. Investigation of the physiological basis for increased exercise threshold for angina pectoris after physical conditioning. I Clin Investig 1974; 54: 736-70.

2 Barry a J, Daly J W, Preutt E d R, Steinmetz J R, BIRKENHEAD N C, RODHAL K. Effects of physical exercise training in patients who have had a myocardial infarction. Am J Cardiol 1966; 17: 1-8.

3 Raffo J A, Luksic I Y, Kappagoda C T, Mary D A S G, Whitaker W, LONDEN R J. Effects of physical training on myocardial ischemia in patients with coronary artery diseasc. Br Heart J 1980; 43: 262-269.
4 Folkins C H, Amsterdam E A. Control and modification of stress emotions through chronic exercise. In: AmsterdaM E A, WILMORE J H, Demaria A N (Eds): Exercise in Cardiovascular health and disease. New York. Yorke medical books; 1977: 280-294.

5 Bruce R A, Kusumi F, Hosmer D. Maximal oxygen intake and nomographic assessment of functional aerobic impairment in cardiovascular discase. Am Heart J 1973; 85: 546.

6 McElroy C L, Gissen S A, Fishbein M C. Exercise induced reduction in myocardial infarct size after coronary artery occlusion in the rat. Circulation 1978; 57: 958-962. 
7 Eckstein $\mathbf{R}$ W. Effect of exercise and coronary artery narrowing on coronary collateral circulation. Cir Res 1957; 5 : 230-5.

8 Heaton W H, Marr K C, Capurro N L. Beneficial effects of physical training on blood flow to the myocardium perfused by chronic collaterals in the exercising dog. Circulation 1978; 57: 575.

9 Astrand P O, Rodhal K. Textbook of Work Physiology. New York. McGraw-Hill 1970.

10 Choquette G, Ferguson R. Blood pressure reduction in "borderline" hypertensives following physical training. Can Med Assoc J. 1973; 108: 699-703.

11 BoYer J L, KASCH F W. Exercise therapy in hypertensive men. JAMA 1970; $211: 1668-71$.
12. Greenberg M A, Arbeit S, Rubin L. The role of physical training in patients with coronary arter $\bar{\Phi}$ disease. Am Heart $J$ 1979; 97: 527-34.

13 Tepperman J. Pearluman D. Effects of exercise an£ anemia on coronary arteries of small animals a을 revealed by the corrosion-cast technique. Circ Re? 1961; 9: 576.

14 Rose G, Prineas R S, Mitchell J R. Myocardiâ infarction and the intrinsic calibre of coronar arteries. $\mathrm{Br}$ Heart $J$ 1967; 29: 548.

15 Froelicher V, Jensen D, Atwood J E, McKirnan M D, Gerber K, Slutsky R, Battler A, Ashburi W, Ross J. Cardiac rehabilitation: evidence fof improvement in myocardial perfusion and functions Arch Phys Med Rehabil 1980; 61: 517-22.

\section{A COURSE ON ANAESTHESIA, ANALGESIA AND SEDATION FOR OUTPATIENT DENTISTRY}

The aforementioned course will be held by the Royal College of Surgeons of England, Faculty of Dental Surgery and Faculty of Anaesthetists from 13th to 17th June, 1983. Further information and application forms are available from Mrs $\mathbf{P}$ M
Neville, Education Secretary, Faculty of Anaesthe tists, Royal College of Surgeons of England, 35!4: Lincoln's Inn Fields, London WC2A 3PN. Telephone 01-405 3474 . 